the gold standard, thoracic US performed quite well. In six of the seven false negative US examinations, chest CT identified malignancy based on parenchymal opacities, adenopathy or the identification of metastatic disease.

Although well conducted, there are several methodological issues that may limit generalisability of the results. First, there was a high incidence of MPE (especially mesothelioma) in this study population. The propensity of mesothelioma to cause thickening of the pleura and diaphragm, as opposed to other malignancies that may cause isolated pleural nodularity, may have biased the performance characteristics of US in this study. This issue will need to be more clearly defined in a larger study with a more typical distribution of MPE not caused by mesothelioma. Second, the US examinations were performed by two experienced radiologists. Although US is used by many non-radiologists to examine the pleural space, the expertise of the authors may have allowed them to identify abnormalities that would have been missed by their non-radiology colleagues. In addition to using a standard $3.5 \mathrm{MHz}$ curvilinear transducer, the authors used a highfrequency 8-15 MHz linear array transducer which has significantly higher resolution. Likewise, the US equipment used in the study is more sophisticated than some of the hand-held units currently used by the non-radiology community. It would have also been interesting if the authors could have confirmed the utility of the "echogenic swirling sign" in their patient population.

One of the largest benefits of US in the evaluation of patients with suspected
MPE is that it is a non-invasive imaging modality that does not expose the patient to radiation. As such, US should be positioned after the clinical examination or after a standard chest radiograph in the algorithm of evaluating patients with pleural effusions. Performing chest US prior to obtaining a chest CT scan will provide the physician with valuable clinical information including the ability to narrow one differential diagnosis, guide pleural drainage and potentially predict the ability of the lung to fully re-expand. Draining the effusion before the chest CT scan will reduce the effects of compressive atelectasis and aid in the evaluation of the underlying parenchyma. A contrary argument, however, is that the presence of pleural fluid improves the sensitivity of the chest CT scan for evaluating pleural thickening and nodularity. Likewise, chest CT scanning has the obvious benefit of identifying parenchymal opacities invisible to US and can simultaneously provide information about mediastinal lymph nodes and extrathoracic disease.

One of the lessons the reader should take home from this study is that chest ultrasonography should proceed in a standardised fashion with attention to and documentation of specific details (see online supplement to paper by Qureshi et $\left.a l^{15}\right)$. By using a systematic approach, important details will not be overlooked and the full benefits of pleural US will be realised.

Competing interests: None.

Thorax 2009;64:97-98. doi:10.1136/thx.2008.107292

\section{REFERENCES}

1. Light RW, Macgregor Ml, Luchsinger PC, et al. Pleural effusions: the diagnostic separation of transudates and exudates. Ann Intern Med 1972;77:507-13.

2. Diacon $\mathbf{A H}$, Brutsche MH, Soler M. Accuracy of pleural puncture sites: a prospective comparison of clinical examination with ultrasound. Chest 2003; 123:436-41.

3. American College of Emergency Physicians. Use of ultrasound imaging by emergency physicians. Ann Emerg Med 2001;38:469-70.

4. American College of Surgeons. Ultrasound examinations by surgeons. 1998. http://www.facs. org/fellows info/statements/st-31.html

5. Board of the Faculty of Clinical Radiology. Ultrasound training recommendations for medical and surgical specialties. London: Royal College of Radiology, 2005.

6. Mayo PH, Doelken P. Pleural ultrasonography. Clin Chest Med 2006;27:215-27.

7. Feller-Kopman D. Ultrasound-guided thoracentesis. Chest 2006;129:1709-14.

8. Mayo PH, Goltz HR, Tafreshi M, et al. Safety of ultrasound-guided thoracentesis in patients receiving mechanical ventilation. Chest 2004;125:1059-62.

9. Grogan DR, Irwin RS, Channick R, et al.

Complications associated with thoracentesis. A prospective, randomized study comparing three different methods. Arch Intern Med 1990;150:873-7.

10. Hirsch JH, Rogers JV, Mack LA. Real-time sonography of pleural opacities. AJR Am J Roentgenol 1981;136:297-301.

11. Yang PC, Luh KT, Chang DB, et al. Value of sonography in determining the nature of pleural effusion: analysis of 320 cases. AJR Am J Roentgenol 1992;159:29-33.

12. Chen KY, Liaw YS, Wang HC, et al. Sonographic septation: a useful prognostic indicator of acute thoracic empyema. J Ultrasound Med 2000;19:837-43.

13. Gorg C, Restrepo I, Schwerk WB. Sonography of malignant pleural effusion. Eur Radiol 1997:7:1195-8.

14. Chian CF, Su WL, Soh LH, et al. Echogenic swirling pattern as a predictor of malignant pleural effusions in patients with malignancies. Chest 2004:126:129-34.

15. Qureshi NR, Rahman NM, Gleeson FV. Thoracic ultrasound in the diagnosis of malignant pleural effusion. Thorax 2009:64:139-43.

\title{
Traffic-related air pollution, genetics and asthma development in children
}

\section{Thomas Sandström, ${ }^{1}$ Frank J Kelly ${ }^{2}$}

In recent years, air pollution has increasingly become recognised as a major

\footnotetext{
${ }^{1}$ Department of Respiratory Medicine and Allergy, University Hospital, Umea, Sweden; ${ }^{2}$ Environmental Research Group, School of Biomedical and Health Sciences, King's College, London, UK
}

Correspondence to: Professor T Sandström, Department of Respiratory Medicine and Allergy, University Hospital, Umea SE-901 85, Sweden; thomas.sandstrom@lung.umu.se contributor to adverse health effects. Numerous studies have shown that poor air quality can adversely affect those with respiratory conditions such as asthma and chronic obstructive pulmonary disease and, more recently, cardiovascular conditions such as myocardial infarctions and stroke. ${ }^{1}$ Wherever the location, air pollution has been shown to be associated with deterioration in patients with these conditions, as well as with increased mortality.

In patients with asthma, air pollution increases symptoms, medication use, bronchoconstriction, emergency room admissions and hospitalisations. These effects are linked to pollutants such as ozone, nitrogen dioxide and particulate matter (PM) and, increasingly, the role of traffic-related air pollution has been highlighted. Traffic pollution consists of a complex mixture of particles and gases from gasoline and diesel engines, together with dust from wear of road surfaces, tyres and brakes. The coarse particles from road dust have been clearly associated with worsening of asthma and respiratory symptoms. ${ }^{2}$ Motor engine particles from diesel engines have been linked with worsening of asthma and increased bronchial hyper-responsiveness, 
a hallmark of asthma. ${ }^{3}$ There is also literature suggesting that diesel particles enhance allergen sensitisation in animals and humans and that they differentially affect the airway mucosa in healthy individuals and those with asthma. ${ }^{45}$ While it is generally accepted that air pollutants may trigger the sensitive airways of subjects with asthma via a number of established pathophysiological processes, it has been more controversial to suggest that air pollution directly contributes to the occurrence of asthma.

In a recent issue of Thorax, Salam and coworkers $^{6}$ concluded that certain genetic variants in glutathione S-transferase (GST) and microsomal epoxide hydrolase (EPHX) contribute strongly to the susceptibility and occurrence of asthma in children living near busy roads. Their findings arose from the Californian Children's Health Study in which the same authors earlier showed that PM air pollution in residential areas was associated with impaired lung growth during adolescence. ${ }^{7}$ Furthermore, the same team recently reported that living in close proximity to major roads impaired lung development even more than that seen in those living in residential areas with poor air quality. After taking into account a wide range of socioeconomic factors, they concluded that specific pollutant components in the air in the immediate proximity to traffic are of importance, with diesel exhaust the main focus. ${ }^{8}$ Diesel exhaust consists primarily of extremely high numbers of nanoparticles in nucleation mode around 10-20 nm together with gaseous components which agglomerate to produce particles around $100 \mathrm{~nm}$. The carbon core particles carry polyaromatic organic hydrocarbon (PAH) components on their surface, some of which can cause oxidative stress. ${ }^{9}$ Oxidative stress with increased consumption of antioxidants has been highlighted as a key component in asthma, ${ }^{10}$ and polymorphisms in GST have been indicated to play a role in this condition. ${ }^{11}$ Further, there is evidence that, in patients with asthma, the epithelium is deficient in its ability to neutralise oxidant attack through a decrease in antioxidant enzymes such as superoxide dismutase and glutathione peroxidase. ${ }^{12}$

Given this background, it is interesting that Salam and coworkers have addressed the role of GST M1, P1 and T1, which not only represent defensive components for limiting oxidative stress but also, together with EPHX, play an important role in the metabolism of PAHs. GSTs detoxify PAHs through the formation of glutathione conjugates. Through the trans-dehydrodiol pathways, reactive semi-quinones are generated which may result in the generation of reactive oxygen species. EPHX1 is of importance in the metabolism of reactive epoxides from activated PAHs.

Salam and coworkers showed that high EPHX1 activity increased the risk of lifetime asthma, but that this was dependent on the GSTP1 variant with the Ile105VAL genotype and, furthermore, residential proximity to major roads. Interestingly, children with GSTP1 105Val/Val genotype and high EPHX1 phenotype had a several fold increase in lifetime asthma compared with those with a low to intermediate phenotype. These findings are important since they suggest plausible mechanisms whereby constituents of incomplete combustion of fossil fuels in motor engines in general - and diesel engines in particularmay adversely affect the delicate cells lining the respiratory tract. It also draws attention to the fact that reactive PAH-derived components such as quinones are of importance, as recently indicated by $\mathrm{Nel} .{ }^{13}$

The study by Salam et al highlights the importance of attaining a better understanding of the traffic-related air pollution components responsible for specific health effects. An increased understanding of the chemistry, metabolism and biological consequences of PAHs is warranted, as are the roles for engine types, fuels, exhaust gases and particles, as well as exhaust filters. The knowledge of how primary emissions influence the air at and near busy roads and how they change further away from major traffic is important to evaluate.

The toxicity of tobacco smoke components is also dependent on GSTs, and Salam and coworkers appreciated that exposure to environmental tobacco smoke (ETS) could have influenced their study outcome. To address this important issue, the authors explored ETS and a range of socioeconomic factors to determine if they could have modified the outcome. Adjustments for socioeconomic and demographic factors, community of residence, maternal smoking during pregnancy and smoking at home were examined, as well as restrictions to analysis in children with health insurance with similar results. The authors recognised that complementary studies are needed to fully dissect the interaction between ETS in relation to pollutants in ambient air.
In conclusion, the study by Salam et al indicates a role for genetic variants in GST and microsomal EPHX1 in detoxifying PAHs from traffic and together to ascertain the susceptibility and occurrence of childhood asthma. It also highlights a lack of detailed knowledge of how air pollutant components differ near heavy trafficked roads and how these vary at increasing distances from roads. It also reminds us that we understand little about the contributions of diesel and gasoline exhaust components to the toxicity of traffic pollution. The fusion of a well-designed epidemiological asthma study with the mechanistic approach to genetic variation in enzymes of $\mathrm{PAH}$ detoxification and oxidative stress places this paper in the forefront of asthma and air pollution research.

Competing interests: None.

Thorax 2009;64:98-99. doi:10.1136/thx.2007.084814

\section{REFERENCES}

1. World Health Organization. Air quality guideline: global update 2005. Geneva: World Health Organization, 2006

2. Brunekreef B, Forsberg B. Epidemiological evidence of effects of coarse airborne particles on health. Eur Respir J 2005;2:309-18.

3. Nordenhäll C, Pourazar J, Ledin MC, et al. Diesel exhaust enhances airway responsiveness in asthmatic subjects. Eur Respir J 2001;5:909-15.

4. Pourazar J, Frew AJ, Blomberg A, et al. Diesel exhaust exposure enhances the expression of IL-13 in the bronchial epithelium of healthy subjects. Respir Med 2004;98:821-5

5. Stenfors N, Nordenhall C, Salvi SS, et al. Different airway inflammatory response in asthmatic and healthy subjects by diesel exhaust. Eur Respir J 2004;23:82-6.

6. Salam MY, Lin P-L, Avol EL, et al. Microsomal epoxide hydrolase, glutathione S-transferase P1, traffic and childhood asthma. Thorax 2007;62:1050-7.

7. Gauderman WJ, Avol E, Gilliland F, et al. The effect of air pollution on lung development from 10 to 18 years of age. N Engl J Med 2004;351:1057-67.

8. Gauderman WJ, Vora H, McConnell R, et al. Effect of exposure to traffic on lung development from 10 to 18 years of age: a cohort study. Lancet 2007:369:571-7.

9. Xia T, Paavo Korge, James N Weiss, et al. Quinones and aromatic chemical compounds in particulate matter induce mitochondrial dysfunction: implications for ultrafine particle toxicity. Environ Health Perspect 2004;112:1347-58.

10. Kelly FJ, Mudway I, Blomberg A, et al. Altered lung antioxidant status in patients with mild asthma. Lancet 1999;354:482-3.

11. Gilliland FD, Li YF, Gong H Jr, et al. Glutathione s-transferases $\mathrm{M} 1$ and $\mathrm{P} 1$ prevent aggravation of allergic responses by secondhand smoke. Am J Respir Crit Care Med 2006;174:1335-41.

12. Comhair SA, Xu W, Ghosh S, et al. Superoxide dismutase inactibvation in pathophysiology of asthmatic airway remodeling andreactivity. Am J Pathol 2005;166:663-74.

13. Nel A. Air pollution-related illness: effects of particles. Science 2005;308:804-6. 
3. Griffiths TL, Burr ML, Campbell IA, et al. Results at 1 year of outpatient multidisciplinary pulmonary rehabilitation: a randomised controlled trial. Lancet 2000;355:362-8.

4. Hicks A, Albert P, Ford V, et al. Limitation to characterizing daily activity in COPD (abstract). Am J Respir Crit Care Med 2008:177:A147.

\section{Non-atopic persistent asthma in children}

Henderson and colleagues ${ }^{1}$ interestingly describe six different wheezing phenotypes among which persistent wheeze is, they say, less associated with atopy than intermediate or late-onset wheeze (but with similar lung function deficits, suggesting a mixture of structural airway abnormalities and atopic wheeze).

We would like to emphasise the fact that children with persistent asthma without allergic sensitisation (ie, non-atopic persistent asthma (NAPA)) constitute a phenotype of its own that should be accounted for separately, since its clinical features differ noticeably from atopic persistent asthma (APA).

At our reference centre for paediatric asthma in a north-eastern region of Italy, there were 14 patients with NAPA out of 1280 seen in the last 5 years (1\%). In this series, $12 / 14$ patients with NAPA (84.7\%) had clinical features of moderate and severe persistent asthma vs 304/1266 patients with APA $(24 \%, p<0.001) ; 8 / 14$ patients with NAPA $(57 \%)$ required hospital admission compared with 130 patients with APA $(10 \%$, $p<0.001)$. The transition from first wheezing (usually viral) and persistent asthma symptoms was much faster in patients with NAPA than in those with APA (mean (SD) 0.5 (0.8) years vs $3.6(2.4)$ years; $p=0.001)$. Moreover, only one of the patients with NAPA had a clinical history of atopic dermatitis compared with 785 (63\%) of those with APA.

Just as children with APA and adults with "intrinsic" asthma, children with NAPA do have intense eosinophilic inflammation of the respiratory airways. ${ }^{1}$ In agreement with this finding, inhaled steroids were an effective treatment in our patients.

Despite its low incidence, this infrequent-although not very rare-paediatric asthma phenotype should not be missed in large epidemiological cohort studies. Nothing is known about which treatment is best for NAPA and-even more importantly- nothing is known about the natural history of this severe disease.

\section{G Longo, E Panontin, G Ventura}

Institute of Child Health, IRCCS Burlo Garofolo, University of Trieste, Trieste, Italy

Correspondence to: Dr G Ventura, Institute of Child Health, IRCCS Burlo Garofolo, University of Trieste, Trieste 34137, Italy; gioviventura@gmail.com

Competing interests: None.

\section{REFERENCES}

1. Henderson J, Granell R, Heron J, et al. Associations of wheezing phenotypes in the first six years of life with atopy, lung function and airway responsiveness in mid childhood. Thorax 2008:63:974-80.

2. Turato G, Barbato A, Baraldo S, et al. Nonatopic children with multitrigger wheezing have airway pathology comparable to atopic asthma. Am J Respir Crit Care Med 2008;178:476-82.

\section{Authors' reply}

We thank Dr Ventura and colleagues for their interest in our paper and for drawing attention to the phenomenon of non-atopic persistent asthma (NAPA). We agree that non-atopic airway inflammation is a potential mechanism for modification of early life influences on airway development. In our paper we highlighted the strong positive association of persistent wheeze with atopy but, as shown in table 2, the prevalence of atopy in this group was $42 \%$. As persistent wheeze comprised $7 \%$ of the total population, non-atopic persistent wheeze would be expected to occur in around $4 \%$ of this unselected population-based sample. This contrasts with the prevalence of NAPA of $1 \%$ reported by Ventura and colleagues from their specialist clinic population in Italy and raises important questions about comparing results from different population samples. It is not clear from their letter how Ventura and colleagues defined persistent asthma but, without frequent early life measures, it would be difficult to disentangle intermediate onset (62\% atopic) from persistent wheeze in our model. As both these phenotypes were very strongly associated with persistent wheezing and with physiciandiagnosed asthma in later childhood, such misclassification could alter the inferences of their respective associations with objective markers of atopy and airway function. Ventura et al make some interesting observations about the early life course of NAPA which are highly relevant to our attempts to disentangle the course of early childhood wheezing trajectories. Clearly, there are more complexities to emerge from approaches to defining the various subphenotypes of asthma, and detection of rare phenotypes - even in relatively large population samples-remains a challenge. The trade-off between population size and the intensity of detailed phenotyping with relevant biomarkers that is feasible in very large epidemiological surveys exemplifies some of these difficulties, although the application of non-invasive markers of inflammation in this setting shows some promise. ${ }^{2}$ There is a need to understand the variation of phenotypes within asthma and how these relate to clinical and pathological end points. A greater understanding of genetic determinants of components of the asthma phenotype, ${ }^{3}$ harmonisation of outcomes between studies and exploration of environmental interactions with genetic variants will hopefully reveal modifiable targets for disease treatment and prevention.

\section{J Henderson, R Granell, J Sterne}

Avon Longitudinal Study of Parents and Children (ALSPAC), University of Bristol, Bristol, UK

Correspondence to: Dr J Henderson, ALSPAC, University of Bristol, Bristol BS8 2BN, UK; a.j.henderson@bris.ac.uk

Competing interests: None.

\section{REFERENCES}

1. Henderson J, Granell R, Heron J, et al. Associations of wheezing phenotypes in the first six years of life with atopy, lung function and airway responsiveness in mid childhood. Thorax 2008;63:974-80.

2. Nicolaou NC, Lowe LA, Murray CS, et al. Exhaled breath condensate $\mathrm{pH}$ and childhood asthma: unselected birth cohort study. Am J Respir Crit Care Med 2006;174:254-9.

3. Bisgaard H, Bonnelykke K, Sleiman PM, et al. Chromosome 17q21 gene variants are associated with asthma and exacerbations but not atopy in early childhood. Am J Respir Crit Care Med 2009;179:179-85.

\section{CORRECTION}

doi:10.1136/thx.2007.084814corr1

T Sandström, F J Kelly. Traffic-related air pollution, genetics and asthma development in children. Thorax 2009;64:98-99. Reference 6 in this editorial is incorrect, it should be Salam MT, Lin P-L, Avol EL, et al. Microsomal epoxide hydrolase, glutathione S-transferase P1, traffic and childhood asthma. Thorax 2007;62:1050-7. 